wenn ihr Ausgangsgehalt aus unbekannten Gründen stark gesenkt ist ${ }^{10}$.

Es sei noch erwähnt, daß sich bestrahlte kernhaltige Zellen über 16 Tage hinsichtlich RNS und löslichen Proteinen zwar wie unbestrahlte kernlose
Zellen verhalten, indem bei weitgehend unverändertem RNS-Spiegel eine Synthese von Proteinen möglich ist, nach den vorstehenden Erörterungen jedoch keine Notwendigkeit vorliegt, hieraus etwa auf gleiche Ursachen zu schließen.

\title{
Untersuchungen über die Verteilung und Reaktionsfähigkeit von Sulfhydrylgruppen in der tierischen Zelle
}

\author{
Von Hanna Ernst und Ulrich Hagen \\ Aus der Biophysikalischen Abteilung des Heiligenberg-Instituts, Heiligenberg, Baden \\ (Dir. Professor Dr. H. Langendorff) \\ (Z. Naturforschg. 14 b, $104-110$ [1959]; eingegangen am 6. August 1958)
}

\begin{abstract}
Rattenleber-Homogenat wurde durch fraktioniertes Zentrifugieren in Mitochondrien, Mikrosomen, Überstehendes und Zellkerne, die ihrerseits in vier Proteinfraktionen zerlegt wurden, aufgeteilt. Die darin enthaltenen Sulfhydrylgruppen wurden durch ampèrometrische $\mathrm{Hg}$-Titration bestimmt. Alkoholdenaturierung der Fraktionen erbrachte überall einen Anstieg der SH (maskierte SH-Gruppen), Denaturierung mit Harnstoffderivaten, Duponol und Enteiweißungsmitteln verminderten meist die meß. baren SH-Gruppen. Es konnte bewiesen werden, daß sich organische Säuren (SSS, TES, MPS) nicht zur Bestimmung von Nichtprotein-SH in tierischem Gewebe eignen.

Der SH-Gehalt der Leberzelle liegt zu $60 \%$ im Kern. Dort wurden im DNS-Protein keine, im RNS.Protein erhebliche Mengen von maskierten SH-Gruppen festgestellt. Diese Proteine enthalten fünfmal soviel Sulfhydrylgruppen pro Stickstoffeinheit wie die übrigen Zellfraktionen.
\end{abstract}

Kürzlich diskutierten wir ausführlich die Ansich. ten, die in der Literatur über den Wirkungsmechanismus der Sulfhydryl-Verbindungen als Strahlenschutzmittel bestehen ${ }^{1}$. Hierbei ergab sich, daß der von Eldjarn und PinL ${ }^{2-4}$ vorgeschlagene Schutzmechanismus die größte Wahrscheinlichkeit besitzt. Die schützenden SH-Verbindungen verbinden sich mit ganz bestimmten SH-Gruppen der Zelle in Form eines gemischten Disulfides und lösen dadurch den Strahlenschutz aus. Einen weiteren Anhalt für einen recht spezifischen Angriffspunkt der SH-Verbindungen geben die autoradiographischen Untersuchungen mit ${ }^{35}$ S-Cystein von Passalacqua und $\mathrm{Koch}^{5}$. Nach diesen ist in Milz und Leber die Aktivität zum größten Teil an den Zellkern gebunden.

Weitere Aussagen über einen möglichen Schutzmechanismus sind nach diesen Vorstellungen nur dann möglich, wenn man versucht, Näheres über die Verteilung und Reaktionsfähigkeit der SH-Gruppen in der Zelle auszusagen. Im Gegensatz zu den zahlreichen Messungen von SH-Gruppen an definierten Proteinen, fehlen unseres Wissens in der Literatur

1 U. Hagen u. R. Koch, Z. Naturforschg. 12 b, 240 [1957].

2 L. Eldjarn and A. Pinl, Progr. Radiobiology, p. 249. Oliver and Boyd, London 1956.

${ }^{3}$ L. Eldjarn u. A. Pihl, J. biol. Chemistry 223, 341 [1956].
Angaben über die Verteilung der SH-Gruppen in der Zelle, welche eine Aufstellung der Gesamt-SHBilanz möglich machen. Meistens wurden nur die SH-Gruppen nach Enteiweißung der Gewebehomogenate analysiert. Im folgenden soll eine Methode geschildert werden, mit der wir versuchen, möglichst die gesamten SH-Gruppen der Zelle zu bestimmen. Die sich daraus ergebenden strahlenbiologischen Fragestellungen sollen in späteren Mitteilungen behandelt werden.

\section{Material und Methoden}

$$
\text { Versuchsmaterial }
$$

Wir verwenden Rattenleber, da sie sich - im Gegensatz zur Milz - leicht entbluten läßt. So konnte der hohe SH-Gehalt des Blutes ${ }^{6}$ sich nicht störend bemerkbar machen. Die übrigen Organe entfallen für unsere Zwecke zunächst wegen ihrer geringen Größe. Wir verwenden männliche Ratten des institutseigenen Inzuchtstammes im Gewicht von $120-150 \mathrm{~g}$. Die Tiere erhalten als Futter „Latz-Rattenkekse“ und Wasser ad libitum. Alle Untersuchungen werden morgens durchgeführt. Auf diese Weise werden Fehlerquellen durch die veränderte

${ }^{4}$ L. Eldjarn u. A. Pihl, J. Amer. chem. Soc. 79, 4589 [1957].

5 F. Passalacqua u. R. Koch, Strahlentherapie 105, 271 [1958].

6 E. del Pianto u. P. Silvestroni, Ricerca sci. 24, 3 [1954]. 
Stoffwechsellage der Leber zu verschiedenen Tageszeiten vermieden 7,8 . Eine 12-stdg. Futterkarenz der Tiere bewirkte keine Änderung der von uns gemessenen SHWerte, wie insgesamt 6 Bilanzversuche ergaben. Zudem besteht auch bei hungernden Tieren eine relativ große Stabilität in der Zusammensetzung der Zellkernproteine $^{\mathbf{9}}$, also in der Fraktion, die uns hier am meisten interessierte.

Zur Entblutung der Leber wird nach Äthernarkose des Tieres und Durchtrennung der Vena cava inf. durch die Aorta descendens $30-50 \mathrm{ml}$ eiskalte $0,25-\mathrm{m}$. Sucrose gespült. Hierdurch kühlt sich die Leber sehr rasch auf etwa $+10^{\circ} \mathrm{C}$ ab. Das Gewebe wurde nach Entfernen der großen Leberstämme bei $0^{\circ}$ weiter verarbeitet.

Fraktionierung und Subfraktionierung

$10 \mathrm{~g}$ Leber - von 2 Tieren - werden mit $50 \mathrm{ml}$ 0,25-m. Sucrose bei $0^{\circ}$ im Glashomogenisator homogenisiert. Die Zellbestandteile werden durch fraktioniertes Zentrifugieren nach Schneider ${ }^{10}$ in Kerne (A), Mitochondrien (B), Mikrosomen (C) und "Überstehendes "“ (D) getrennt. Die Kerne werden vor den Waschungen durch ein Perlonnetz filtriert. Die vereinigten Waschwässer werden in den SH-Messungen als eigene Fraktion behandelt und dann den Meßwerten des „Überstehenden", dem Zellplasma zugerechnet. Eine lichtmikroskopische Kontrolle der Zellfraktionen zeigte bei Zellkernen und Mitochondrien eine befriedigende Reinheit.

Als „Subfraktionierung“ bezeichnen wir im folgenden die weitere Auftrennung von Zellbestandteilen in einzelne Proteine.

Die Subfraktionierung der Zellkerne erfolgt im wesentlichen nach dem von WeInmanN ${ }^{11}$ angegebenen Schema. Die Zellkernfraktion wird 1-mal mit Sucrose und 2-mal mit $0,9 \% \mathrm{NaCl}$ hochtourig gewaschen; die Waschwässer bilden die Subfraktion I. Die Extraktion der Nucleoproteide wird mit $1-m$. NaCl ${ }^{12}$ (10 min bei $0^{\circ}$, durchgeführt. Nach Abzentrifugieren (5 min $20000 \mathrm{~g}$ ) wird aus dem Überstand das Nucleoproteid durch Verdünnen auf 0,14-m. NaCl ausgefällt. Die Fällung wird mit $0,1-n$. NaOH wieder gelöst und der SH-Gehalt sofort bestimmt (Subfraktion III). Aus dieser Fraktion löste Weinmann ${ }^{11}$ mit $0,1-n . \mathrm{H}_{2} \mathrm{SO}_{4}$ noch den Histonanteil heraus. Wir fanden in ihm keine SH-Verbindungen und unterließen später diese Extraktion.

Aus dem Sediment der NaCl-Extraktion läßt sich mit 0,1- $n$. NaOH eine Eiweißfraktion gewinnen, die Sulfhydryle enthält (Subfraktion II). Nach der Lage ihres

7 L. v. Beck, V. D. Rieck u. B. Duncan, Proc. Soc. exp. Biol. Med. 97, 229 [1958].

8 J. W. Goldzieher, W. B. Rawls u. M. A. Goldzieher, J. biol. Chemistry 203, 519 [1953].

9 C. Allard, G. de Lamiranda u. A. Cantero, Exp. Cell Res. 13, 69 [1957].

10 W. C. Schneider, J. biol. Chemistry 176, 259 [1948].

11 W. D. Weinmann, Protoplasma 47, 259 [1956].

12 A. E. Mirsky u. A. W. Pollister, J. gen. Physiol. 30, 117 [1946].

13 Z. Dische u. K. Schwarz, Mikrochim. Acta [Wien] 2, 13 [1937]. isoelektrischen Punktes bezeichnet sie WeInmann als „saures Protein“ und charakterisiert sie als Phosphoproteid. Der verbleibende kleine Rest der Zellkerne aus Lipoproteinen und Phosphatiden wird mit 1-n. $\mathrm{NaOH}$ extrahiert (Subfraktion IV). Auch darin sind meßbare Mengen an SH-Gruppen enthalten. Die zurückbleibenden Reste der Kerne erwiesen sich sulfhydrylfrei.

Nach qualitativen Bestimmungen von Desoxyribonucleinsäure (DNS) und Ribonucleinsäure (RNS) in den einzelnen Proteinen (Methode nach Dische ${ }^{13}$ und $\mathrm{W}_{\mathrm{EBB}}{ }^{14}$, liegt die gesamte DNS in der Subfraktion III vor; wir bezeichnen sie deshalb hier als DNS-Protein. Daneben enthält sie etwa $20 \%$ der vorhandenen RNS. Die übrige RNS findet sich in Subfraktion II, hier als RNS-Protein bezeichnet. Waschwässer (I) und Membranproteine (IV) sind frei von Nucleinsäuren. Es sei darauf hingewiesen, daß die Subfraktionen II und III neben den Nucleoproteinen zweifellos noch andere Eiweißverbindungen enthalten. Doch läßt sich der unterschiedliche Gehalt an RNS oder DNS gut zur Charakterisierung der Extrakte heranziehen.

Eine Subfraktionierung der Mitochondrien nach Kielly ${ }^{15}$ oder der Mikrosomen nach Hultin ${ }^{16}$ erbrachte keine Zunahme der meßbaren SH-Gruppen; sie war für unsere Bilanzbetrachtungen deshalb auch nicht notwendig. Eine Übersicht über den gesamten Trennungsgang gibt die Tab. 1.

Von allen Fraktionen und Subfraktionen werden $0,5 \mathrm{ml}$ zur Stickstoffbestimmung in Conway-Zellen ${ }^{17}$ verwendet. Die Fraktionen wurden gelegentlich papierchromatographisch auf ihren Gehalt an niedermolekularen SH-Körpern sowie freien Aminosäuren überprüft.

S u lf h y d r y l - B e s t i m mung

Trotz der großen Zahl der in der Literatur beschriebenen Sulfhydryl-Nachweise ${ }^{\mathbf{1 8}, 19}$ konnten wir nur die ampèrometrische Titrationsmethode verwenden. Sie erlaubt verläßliche SH-Messungen auch im nativen Eiweiß. Die meisten übrigen Methoden zur SH-Messung im Eiweiß verlangen eine optisch klare Lösung, was jedoch bei den Zellfraktionen nicht zu verwirklichen ist.

Bei der ampèrometrischen Titration reagiert die $\mathrm{SH}$ Gruppe mit dem zugegebenen $\mathrm{Ag}^{\oplus}$ oder $\mathrm{Hg}^{2 \oplus}$-Ion unter Mercaptidbildung. Der Endpunkt des SchwermetallVerbrauches wird durch das Auftreten eines Diffusionsstromes zwischen zwei Elektroden angezeigt. Wir verwenden $0,05-m$. $\mathrm{HgCl}_{2}$ als Titrationslösung, da es spezifischer als $\mathrm{AgNO}_{3}$ reagiert ${ }^{20,21}$. Im allgemeinen bindet ein $\mathrm{Hg}^{2}{ }^{\oplus}$-Ion eine Sulfhydrylgruppe, $1 \mu \mathrm{Mol} \mathrm{SH}(=33 \gamma$ $\mathrm{SH})$ entspricht dann 0,02 ml 0,05-m. $\mathrm{HgCl}_{2}$. Bei

\footnotetext{
14 J. M. Webв u. H. B. Levy, J. biol. Chemistry 213, 107 [1955].

15 R. K. Kielly, Biochim. biophysica Acta [Amsterdam] 21, 574 [1956].

16 T. Hultin, Exp. Cell Res. 12, 290 [1957].

17 H. Wüst, Klin. Wschr. 33, 185 [1955].

18 J. W. Patterson u. A. Lazarow, in „Glutathione“, Academic Press, Inc. New York 1954.

19 K. Heide, Behringwerk-Mitteilungen 30 [1955].

20 R. E. Bennesch, H. A. Lardy, R. Bennesch, J. biol. Chemistry 216, 663 [1955].

21 V. M. Ingram, Biochem. J. 59, 653 [1955].
} 
Leberhomogenat

Kerne (A)
1 Waschung mit Sucrose
2 Waschungen mit 0,14-m. NaCl

Tab. 1. Schema der Gewebefraktionierung.

günstiger sterischer Anordnung der Sulfhydryle im Protein kann das $\mathrm{Hg}^{2 \oplus}$-Ion aber auch zwei SH-Gruppen binden ${ }^{21-23}$. So finden wir bei der Titration niedermolekularer SH-Körper meistens ein höheres Bindungsvermögen des Quecksilbers als stöchiometrisch zu erwarten ist. Zur Berechnung der SH-Menge in den Zellfraktionen müssen wir jedoch zunächst eine stöchiometrische Reaktion im Verhältnis $1: 1$ zum verbrauchten $\mathrm{Hg}^{2 \oplus}$-Ion annehmen, da Einzelheiten der Reaktion des Hg mit den Protein-SH-Gruppen noch nicht bekannt sind.

Die Titration wurde im KCl-Phosphatpuffer $\left(p_{\mathrm{H}} 7,4\right)^{24,25}$ im Stickstoffstrom durchgeführt (Doppelbestimmung). Die Pufferkapazität reichte aus, um $5 \mathrm{ml}$ 0,1- $n$. NaOH-Extrakt in neutralem Bereich zu halten. Der 1-n. NaOH-Auszug (Subfraktion IV) mußte nach Pufferzugabe noch etwas angesäuert werden. Bei der Bestimmung der Eichkurven mit niedermolekularen SHKörpern setzten wir nach Bennesch und Mitarbb. ${ }^{20}$ 0,05\% Gelatine zu, um eine Vergiftung der Platinelektrode durch die Eichsubstanz zu verlangsamen.

\section{S H - M essungen im Gewebe}

Bei der ampèrometrischen SH-Messung an wohldefinierten Proteinen werden schon im nativen Zustand sehr unterschiedliche Werte gemessen ${ }^{21}$; einwandfrei reproduzierbare Bestimmungen gelingen erst nach Denaturierung der Proteine, die zudem eine deutliche $\mathrm{Zu}$ nahme der meßbaren SH-Gruppen bringt. Diese Verhältnisse waren bei unserem eigenen heterogenen Material besonders zu beachten.

24 J. M. Kolthoff, W. Stricks u. L. Morren, Analytic Chem. 26, 366 [1954].

25 V. M. Ingram, Biochem. J. 65, 760 [1957].

\section{Ergebnisse}

Im folgenden möchten wir nach der Nomenklatur von BARron ${ }^{26}$ als freie SH-Gruppen diejenigen SHMengen bezeichnen, die in der unveränderten Suspension sofort nach Extraktion meßbar sind. Die maskierten SH-Gruppen werden nach irreversibler Auffaltung der Protein-Kettenmoleküle durch organische Lösungsmittel frei. Die trägen SH-Gruppen Barrons haben wir nicht untersucht. Bei unseren Bilanzversuchen bestimmten wir neben den freien

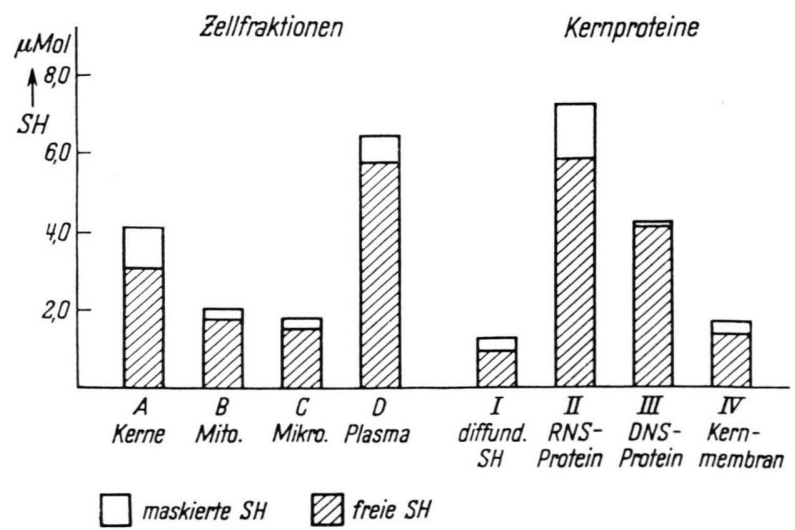

Abb. 1. Verteilung der SH-Gruppen in der Rattenleber.

22 L. A. Ä. Sluyterman, Biochim. biophysica Acta [Amsterdam] 25, 402 [1957].

23 M. Murayama, J. biol. Chemistry 228, 231 [1957].

26 E. S. G. Barron, Advances in Enzymol. 11, 201 [1951]. 


\begin{tabular}{|c|c|c|c|c|c|c|}
\hline \multirow{2}{*}{ Fraktion } & \multirow{2}{*}{$\begin{array}{c}\text { Zahl der } \\
\text { Versuche }\end{array}$} & \multicolumn{2}{|c|}{$\begin{array}{c}\text { MMol SH/g Leber } \\
\text { (Feuchtgewicht) }\end{array}$} & $\begin{array}{c}\text { mg N/g } \\
\text { Leber }\end{array}$ & \multicolumn{2}{c|}{$\mu \mathrm{Mol} \mathrm{SH} / \mathrm{mg} \mathrm{N}$} \\
\cline { 3 - 7 } & & frei & maskiert & & frei & maskiert \\
\hline A & 16 & $3,05 \pm 0,7$ & $4,12 \pm 0,1$ & $6,5 \pm 1,33$ & $0,52 \pm 0,13$ & $0,67 \pm 0,14$ \\
B & 10 & $1,81 \pm 0,26$ & $2,05 \pm 0,24$ & $4,06 \pm 0,33$ & $0,39 \pm 0,04$ & $0,47 \pm 0,11$ \\
C & 12 & $1,54 \pm 0,13$ & $1,88 \pm 0,24$ & $4,00 \pm 0,26$ & $0,38 \pm 0,026$ & $0,45 \pm 0,046$ \\
D & 11 & $5,8 \pm 0,38$ & $6,42 \pm 0,36$ & $8,13 \pm 0,49$ & $0,74 \pm 0,052$ & $0,83 \pm 0,065$ \\
I & 10 & $1,00 \pm 0,17$ & $1,28 \pm 0,145$ & $1,86 \pm 0,05$ & $0,64 \pm 0,22$ & $0,64 \pm 0,14$ \\
II & 5 & $5,89 \pm 0,11$ & $7,33 \pm 0,67$ & $2,33 \pm 0,22$ & $2,10 \pm 0,44$ & $2,58 \pm 0,27$ \\
III & 11 & $4,14 \pm 0,56$ & $4,21 \pm 0,47$ & $1,17 \pm 0,14$ & $3,54 \pm 0,5$ & $3,10 \pm 0,44$ \\
IV & 5 & $1,45 \pm 0,10$ & $1,73 \pm 0,25$ & $1,10 \pm 0,11$ & $1,3 \pm 0,07$ & $1,65 \pm 0,37$ \\
\hline
\end{tabular}

Tab. 2. Verteilung der SH-Gruppen in der Leberzelle.

\begin{tabular}{|c|c|c|c|}
\hline Autor & $\mu \mathrm{Mol} \mathrm{SH} / \mathrm{mg} \mathrm{N}$ & Protein & Bestimmungs-Methode \\
\hline Lontie u. Beckers 27 & 0,037 & Human-Albumin & amp. $\mathrm{AgNO}_{3}$-Titration \\
\hline Lontie u. Beckers 28 & 0,67 & Eier-Albumin & amp. $\mathrm{AgNO}_{3}$-Titration \\
\hline $\begin{array}{l}\text { Hommes, Dozy u. } \\
\text { Huisman } 29\end{array}$ & 0,67 & Haemoglobin & amp. $\mathrm{AgNO}_{3}$-Titration \\
\hline Nishiyama 30 & $0,79\left(\frac{\mathrm{Mol}}{\mathrm{mg} \mathrm{N}}\right)$ & Ribonucleoprotein & Ferricyanid \\
\hline
\end{tabular}

Tab. 3. Sulfhydrylgehalt einiger definierter Proteine.

SH-Gruppen die maskierten SH-Gruppen nach Denaturierung mit Äthylalkohol (50\% Endkonzentration).

In der Abb. 1 sind unsere Meßwerte an freien und maskierten SH-Gruppen dargestellt. Der mittlere Fehler dieser Messungen findet sich in Tab. 2, in ihr ist auch der Stickstoffgehalt der Fraktionen angegeben und der SH-Gehalt auf ihn bezogen.

Aus der Abb. 1 wird deutlich, daß der größte Teil der Sulfhydrylgruppen im Zellkern zu finden ist. In der Suspension der intakten Kerne erhält man sehr unterschiedliche Werte, die Streuung des Mittelwertes ist relativ hoch. Zum Teil bestehen technische Schwierigkeiten bei der Messung, da in der Pufferlösung lange Fäden von Nucleoproteinen ausfallen, die die Platinelektrode verlegen und die Meßwerte beeinflussen; außerdem vermögen wohl die $\mathrm{Hg}^{2}{ }^{\oplus}$. Ionen nicht den ganzen Zellkern zu durchdringen* Zerlegt man die Kerne in die einzelnen Subfraktionen, so ist die Summe der gemessenen SH-Gruppen wesentlich höher; sie beträgt $58 \%$ des Gesamt-SH der Zelle. Die beiden anderen Partikelfraktionen, die Mitochondrien und die Mikrosomen, enthalten nur etwa je $1 / 12$ der Gesamt-SH-Gruppen.

Zählen wir den SH-Gehalt der Fraktionen zusammen, so ergibt sich $24,9 \mu$ Mole SH pro g Frischgewicht, das sind $82,1 \mathrm{mg}-\% \mathrm{SH}$. Dieser Wert ist wesentlich höher als der anderer Autoren (14 bis $28 \mathrm{mg}-\%)$; es wird weiter unten noch gezeigt werden, daß diese nur einen Teil der Zell-SH-Gruppen erfaßten.

Eine bessere Charakterisierung des SH-Gehaltes der einzelnen Fraktionen bringt die Berücksichtigung des Stickstoffgehaltes. Aus den Werten der Tab. 2 wird deutlich, daß die Kernproteine II und III die 4-6-fache SH-Konzentration gegenüber den Cytoplasma-Fraktionen aufweisen. Auch das mit 1-n. $\mathrm{NaOH}$ extrahierte Kernmembran-Protein enthält noch mehr SH-Gruppen als das Plasma. In der Subfraktion I und im Überstehenden finden sich papierchromatographisch reichlich niedermolekulare SH-Verbindungen.

Zum Vergleich der einzelnen SH-Konzentrationen $(\mathrm{SH} / \mathrm{mg} \mathrm{N})$ in der Rattenleber seien SulfhydrylBestimmungen in einigen definierten Proteinen herangezogen .

Unsere Cytoplasma-Fraktionen weisen also eine ähnliche SH-Konzentration auf wie Ovalbumin und Hämoglobin. Die von Nishiyama ${ }^{30}$ gemessene $\mathrm{SH}$ Konzentration im RNS-Protein ist mit unseren Werten nur bedingt vergleichbar, da die Methoden verschieden sind.

Zwei Fragen sind für die Deutung der oben geschilderten Messungen zu beachten: 1. Welche Ver-

* Befriedigende Ergebnisse wurden später bei der Messung im Tris-Puffer erzielt. 
luste an SH-Gruppen entstehen während der Aufarbeitung der einzelnen Fraktionen? Hier war besonders die Alkalilabilität der SH-Gruppen bei der Extraktion der Kernproteine zu prüfen. 2. Ist die Denaturierung der Proteine mit Alkohol geeignet, alle „maskierten SH-Gruppen“ zu erfassen oder sind andere Verfahren der Denaturierung oder Auffaltung der Proteinketten geeigneter?

\section{Alkali-Labilität der SH-Gruppen}

Sulfhydrylgruppen werden im allgemeinen (BAR${ }_{\text {RON }}{ }^{26}$ ) in alkalischem Milieu wesentlich rascher oxydiert als in neutralem oder saurem. So waren die in Sucrose suspendierten Zellfraktionen kaum durch Autoxydation gefährdet; 24-stdg. Einfrieren veränderte den erfaßbaren SH-Gehalt nicht. Eine Oxydation war aber bei der Laugeextraktion der Subfraktion II und III zu befürchten.

\begin{tabular}{|l|c|c|c|c|c|c|}
\hline \multirow{2}{*}{ Fraktion } & \multicolumn{5}{|c|}{$0,1-n . \mathrm{NaOH}-20^{\circ} \mathrm{C}$} \\
\cline { 2 - 7 } & sofort & $10^{\prime}$ & $15^{\prime}$ & $20^{\prime}$ & $30^{\prime}$ & $\begin{array}{c}5^{\prime} \\
\text { rühren }\end{array}$ \\
\hline D & 100 & 100 & 80 & & & 50 \\
D $_{N_{2}}$ & 100 & & 85 & & & \\
II & 100 & 80 & 50 & & & \\
IIN $_{N_{2}}$ & 100 & 90 & & 35 & 35 & \\
III $_{\text {III }}$ & 100 & 10 & & & & \\
\hline
\end{tabular}

Tab. 4. Alkali-Labilität der SH-Gruppen (in \% der freien SH).

Entsprechende Versuche (Tab. 4) zeigten, daß die einzelnen Fraktionen unterschiedlich alkalilabil sind. Subfraktion III ist empfindlicher als D oder II. Extrahiert man unter Stickstoff, so wird die Autoxydation von SH-Gruppen verringert, nicht aber unterbunden. Rühren oder Schütteln führt zu besonders starken SH-Verlusten. Dies ließ uns darauf achten, die Kernproteine unmittelbar nach der Laugen- extraktion zu titrieren und unnötigen LuftsauerstoffZutritt in die Lösungen zu vermeiden.

Versuche, die Stabilität der SH-Gruppen durch eine Zugabe von Komplexbildnern als Oxydationsschutz zu erhöhen, mißlangen. Der „SH-Gehalt“ der Fraktionen steigt zwar nach Zugabe von Natriumäthylendiamintetraessigsäure (EDTA) beträchtlich an, doch beruht dies auf der Komplexbindung der titrierten $\mathrm{Hg}^{2 \oplus}$-Ionen. Wir fanden, daß $1 \mathrm{ml}$ 0,1-m. Na-EDTA $0,167 \mathrm{ml}$ 0,05-m. $\mathrm{HgCl}_{2}$ bindet.

\section{SH-Gruppen nach Denaturierung der Proteine}

Neben der Messung der „maskierten“ SH-Gruppen versuchten wir, den SH-Gehalt durch Einwirkung von Harnstoff, Guanidinchlorid, Duponol und verschiedenen Säuren zu verändern.

Alkohol- oder Acetonzugabe (Endkonzentration $50 \%$ ) führt in allen Fraktionen zu einer Zunahme der erfaßbaren SH-Gruppen (Tab. 5). Eine ähnlich konstante und erhöhte SH-Ausbeute beschreiben nach Methanol-Denaturierung IngBar und Kass ${ }^{31}$. Nach Barron ${ }^{26}$ sollen hierdurch die H-Brücken frei und damit versteckt liegende SH-Gruppen für die $\mathrm{Hg}$-Ionen zugänglich werden.

Weitaus am meisten maskierte SH-Gruppen enthält das RNS-Protein. Sie fehlen dagegen im DNSProtein. Daraus ist auch zu entnehmen, daß das sulfhydryl-haltige Eiweiß des RNS-Proteins nur in geringer Konzentration im DNS-Protein als Verunreinigung enthalten sein kann; die beiden SHTräger in den beiden Subfraktionen sind also durch unsere Aufarbeitung gut voneinander getrennt worden. Ergänzend ist zu bemerken, daß das SH-haltige Protein der II. Fraktion nicht in naher Verbindung mit der Ribonucleinsäure stehen kann, denn sonst müßten sich maskierte Sulfhydryle in der III. Kern-

\begin{tabular}{|c|c|c|c|c|c|c|}
\hline \multirow{2}{*}{ Fraktion } & \multirow{2}{*}{ frei $\mathrm{SH}$} & \multirow{2}{*}{$\begin{array}{l}\text { mask. SH } \\
(50 \% \text { Alk.) }\end{array}$} & \multicolumn{4}{|c|}{ SH-Gehalt nach Denaturierung mit } \\
\hline & & & $\begin{array}{c}\text { Harnstoff } \\
(5-m .)\end{array}$ & $\begin{array}{l}\text { Guanidin } \\
\quad(3-m .)\end{array}$ & $\begin{array}{c}\text { Sulfosalicylsäure } \\
(2 \%)\end{array}$ & $\begin{array}{c}\text { Duponol } \\
(2,5 \%)\end{array}$ \\
\hline A & 100 & 159 & & & 100 & \\
\hline B & 100 & 114 & 103 & 100 & 108 & 110 \\
\hline C & 100 & 122 & 100 & 80 & 123 & 100 \\
\hline D & 100 & 111 & 82 & 100 & 41 & 102 \\
\hline I & 100 & 128 & 80 & & 68 & \\
\hline II & 100 & 125 & 54 & & 100 & 76 \\
\hline III & 100 & 102 & 44 & 53 & 100 & 0 \\
\hline IV & 100 & 119 & & & & \\
\hline
\end{tabular}

Tab. 5. SH-Gruppen nach Eiweiß-Denaturierung (in \% der freien SH-Gruppen. $n=4-12$ ). 
fraktion nachweisen lassen. Ähnliches ergibt sich bei der DNS, denn der säure-extrahierte Histonanteil des Desoxyribonucleoproteids erwies sich frei von Sulfhydrylen.

Harnstoff- und Guanidinchlorid-Lösungen öffnen, wie Tab. 5 zeigt, in keiner Fraktion die Eiweißkette soweit, daß alle maskierten SH-Gruppen durch die $\mathrm{Hg}$-Titration erfaßt werden können. In den Subfraktionen II und III sind bedeutend weniger SHGruppen zu messen, vielleicht infolge neuer Faltungen und Verknäuelungen oder durch das alkalische Milieu der Agentien. Diese Beobachtungen stimmen mit denen der Literatur weitgehend überein ${ }^{20,27,32-34}$, nach denen eine Harnstoff-Denaturierung in den verschiedenen Proteinen zu einer stark unterschiedlichen $\mathrm{Zu}$ - oder Abnahme der ampèrometrisch meßbaren SH-Gruppen führt.

Bessere Ausbeuten lassen sich durch Behandeln der Fraktionen mit Duponol (Dodecylsulfat) erzielen ${ }^{35}$. Die von Barron definerten "maskierten SHGruppen" werden nach unseren Messungen aber auch nicht durch Duponol erfaßt.

SH-Gruppen nach Säuredenaturierung der Proteine

Die Behandlung unserer Fraktionen mit verschiedenen Säuren zur Eiweiß-Denaturierung gibt uns die Möglichkeit, die Angaben der Literatur über den SH-Gehalt von tierischem Gewebe mit unseren eige- nen Werten zu vergleichen. Bei den verschiedenen kolorimetrischen SH-Nachweisen fällte man in den Gewebshomogenaten die trübenden Proteine mit Metaphosphorsäure, Trichloressigsäure oder Sulfosalicylsäure aus. Die SH-Gruppen, die so gemessen wurden, bezeichnet man allgemein als "Nicht-Protein-SH“ = NP-SH.

Mißt man ampèrometrisch die trübe Suspension, die aus den einzelnen Fraktionen nach Sulfosalicylsäure-Denaturierung entsteht, so werden die SHGruppen in den Partikelfraktionen nicht wesentlich verändert (Tab. 5). Im Überstehenden (D) und in den Kern-Waschwässern erfolgt sogar eine Abnahme der meßbaren SH-Gruppen.

Um unsere Methode mit den kolorimetrischen Methoden vergleichen zu können, titrierten wir auch den klaren, filtrierten Überstand der mit den genannten Säuren denaturierten Fraktionen sowie eine Aufschwemmung der ausgefällten Eiweiß-Stoffe in Pufferlösung.

Die Verluste an SH-Gruppen, die durch die Säurefällung entstehen, sind augenfällig. Filtrat + Sediment ergeben niemals den SH-Gehalt der Gesamtsuspension oder den Wert der freien SH-Gruppen. Im Filtrat, das für die kolorimetrischen Bestimmungsmethoden verwendet wurde, finden sich größere SH-Mengen nur im Plasma. Die Wirkung der einzelnen Säuren ist zudem sehr unterschiedlich.

In diesem Zusammenhang seien die Messungen verschiedener Autoren an Leberhomogenaten verglichen.

\begin{tabular}{|c|c|c|c|c|c|c|c|c|c|c|c|}
\hline \multirow{2}{*}{ Fraktion } & \multicolumn{2}{|c|}{$\begin{array}{c}\mathrm{SH} \\
\text { aus Tab. } 5\end{array}$} & \multicolumn{3}{|c|}{$\begin{array}{l}\text { Sulfosalicylsäure } \\
2 \% \text { Endkonz. }\end{array}$} & \multicolumn{3}{|c|}{$\begin{array}{l}\text { Metaphosphorsäure } \\
2 \% \text { Endkonz. }\end{array}$} & \multicolumn{3}{|c|}{$\begin{array}{c}\text { Trichloressigsäure } \\
2 \% \text { Endkonz. }\end{array}$} \\
\hline & frei & mask. & $\begin{array}{l}\text { Sus- } \\
\text { pens. }\end{array}$ & $\begin{array}{l}\text { Fil- } \\
\text { trat }\end{array}$ & $\begin{array}{c}\text { Sediment- } \\
\text { Auf- } \\
\text { schwemmg. }\end{array}$ & $\begin{array}{l}\text { Sus- } \\
\text { pens. }\end{array}$ & $\begin{array}{l}\text { Fil- } \\
\text { trat }\end{array}$ & $\begin{array}{l}\text { Sediment- } \\
\text { Auf- } \\
\text { schwemmg. }\end{array}$ & $\begin{array}{l}\text { Sus- } \\
\text { pens. }\end{array}$ & $\begin{array}{l}\text { Fil- } \\
\text { trat }\end{array}$ & $\begin{array}{l}\text { Sediment- } \\
\text { Auf- } \\
\text { schwemmg. }\end{array}$ \\
\hline A & 100 & 159 & 100 & 11 & 8 & & & & & & \\
\hline $\mathrm{B}$ & 100 & 114 & 108 & 0 & 0 & 120 & 50 & 25 & 100 & 33 & 0 \\
\hline C & 100 & 122 & 123 & 17 & 6 & & 33 & 18 & & 10 & 70 \\
\hline D & 100 & 119 & 41 & 25 & 10 & & 90 & 0 & & 30 & 60 \\
\hline II & 100 & 125 & & 10 & 0 & & 0 & 0 & & 0 & 60 \\
\hline III & 100 & 102 & & & & & & & & 0 & 50 \\
\hline
\end{tabular}

Tab. 6. SH-Verteilung in der Leberzelle nach Säure-Denaturierung.

27 R. Lontie u. G. Beckers, J. Indian chem. Soc. 34, 93 [1957].

28 R. Lontie u. G. Beckers, J. Indian chem. Soc. 33, 289 [1956].

29 F. A. Hommes, A. Dozy u. T. H. J. Huisman, Biochem. J. 68, 309 [1958].

30 T. Nishiyama, J. Biochemistry [Tokyo] 42, 627 [1955].

31 S. H. Ingbar u. E. H. Kass, Proc. Soc. exp. Biol. Med. 77, 74 [1951].
32 H. Neurath, J. P, Greenstein, F. W. Putnam u. J. O. Erickson, Chem. Reviews 34, 157 [1944].

33 J. P. Greenstein u. W. V. Jenrette, J. nat. Cancer. Inst. 1, 91 [1940].

${ }^{34}$ C. M. Kay u. J. T. Edsall, Arch. Biochem. Biophysics 65, 354 [1956].

35 M. L. Anson, Science [New York] 90, 256 [1939]. 


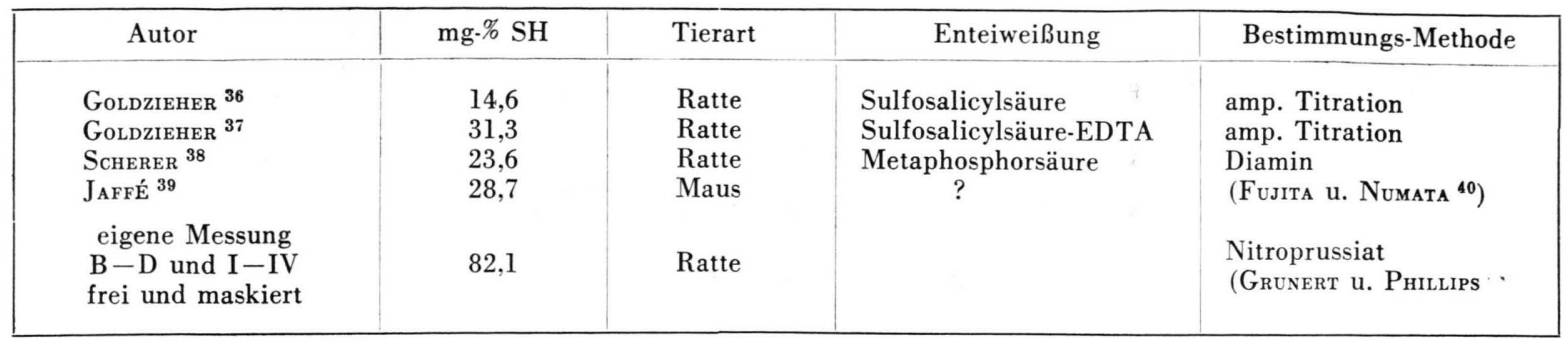

Tab. 7. Nichtprotein-SH (NP-SH) im Lebergewebe.

Die unterschiedlichen Ergebnisse von GoLDZIEHER ${ }^{36}$ und von Scherer ${ }^{38}$ sind nach der Tab. 6 erklärlich. In den Überstand der mit Metaphosphorsäure denaturierten Proteine gehen dreimal soviel SH-Gruppen über als in den SulfosalicylsäureExtrakt. Den wesentlich höheren SH-Wert, den GoLDzieHer $1953^{37}$ gemessen hat, möchten wir nach unseren Erfahrungen auf den Zusatz von EDTA zurückführen.

Auf Grund dieses Vergleiches müssen wir bei allen NP-SH-Messungen in tierischen und pflanz-

36 J. Goldzieher, P. K. Besch u. M. E. Velez, J. biol. Chemistry 231, 445 [1958].

37 J. Goldzieher, W. B. Rawls u. M. A. Goldzieher, Endocrinology 52, 580 [1953].

38 E. Scherer, Klin. Wschr. 34, 95 [1956]. lichen Geweben die erhaltenen Ergebnisse als Plasma-Protein-SH bezeichnen. Dies gilt auch für ampèrometrische Bestimmungen, die im klaren, enteiweißten Filtrat durchgeführt wurden. Unsere oben ausgeführten Meßergebnisse zeigen aber, daß die meisten SH-Gruppen der Zelle sowie die SH-reichsten Proteine im Zellkern zu finden sind. Nicht nur für eine strahlenbiologische, sondern auch für jede biologische Betrachtung des SH-Stoffwechsels, dürfte dieser Reichtum des Zellkerns an SH-Gruppen zu beachten sein.

39 W. G. JaffÉ, Proc. Soc. exp. Biol. Med. 97, 665 [1958].

40 A. Fujita u. J. Numata, Biochem. Z. 300, 264 [1938].

41 Grunert und Phillips, aus: Methods of Biochemical Analysis, Chinard u. Hellerman, p. 21. Interscience Publishers Inc., New York 1954. 